\title{
Pharmacological Topics of Bone Metabolism: The Physiological Function of the Sympathetic Nervous System in Modulating Bone Resorption
}

\author{
Akifumi Togari ${ }^{1}{ }^{*}$ and Michitsugu Arai ${ }^{1}$ \\ ${ }^{1}$ Department of Pharmacology, School of Dentistry, Aichi-Gakuin University, Nagoya 464-8650, Japan
}

Received November 22, 2007; Accepted January 11, 2008

\begin{abstract}
The vertebrate skeleton is richly innervated with adrenergic and peptidergic nerve terminals, and these play important roles in bone remodeling. Recent studies have generally shown that increased sympathetic nervous activity causes bone loss via an increase in bone resorption and a decrease in bone formation. Increased bone resorption is based on the stimulation of both osteoclast formation and osteoclast activity. These effects are associated with $\beta_{2-}$ adrenergic activity toward both osteoblastic and osteoclastic cells. Such findings indicate that $\beta$-blockers may be effective against osteoporosis, in which case there is increased sympathetic activity. This review summarizes evidence obtained both in vitro and in vivo implicating sympathetic neuron action in bone resorption.
\end{abstract}

Keywords: sympathetic nervous system, osteoclastogenesis, bone resorption, osteoporosis, $\beta$-blocker, bone metabolism

\section{Introduction}

Bone remodeling is the physiological process consisting of two balanced opposing activities: the formation of new bone by osteoblasts and the resorption of old bone by osteoclasts. Osteoblastic and osteoclastic activities are regulated by several systemic hormones, including parathyroid hormone (PTH), 1,25-dihydroxyvitamin $\mathrm{D}_{3}$ $\left(1,25(\mathrm{OH})_{2} \mathrm{D}_{3}\right)$, calcitonin, glucocorticoids, sex steroids, and thyroid hormones; and pathological disturbances in the production or activities of these hormones lead to pathological conditions in the skeleton. Moreover, various factors are produced by the bone cells themselves and others are released by non-bone cells in the vicinity. Such autocrine and paracrine factors include cytokines, growth factors, and prostaglandins (PGs). To some extent, the release of these local factors is controlled by the systemic hormones. In addition to endocrine and paracrine/autocrine mechanisms, bone remodelling, like most other homeostatic functions, has also been revealed to be under sympathetic control.

*Corresponding author. togariaf@dpc.aichi-gakuin.ac.jp

Published online in J-STAGE

doi: $10.1254 /$ jphs.FM0070227
In recent studies $(1-5)$, it has become evident that bone cells are equipped with functional receptors for several neuro-osteogenic factors; and, therefore, it has been proposed that signaling molecules in the nervous system may participate in the control of bone metabolism and that consequently a neuro-osteogenic network may exist, similar to the previously proposed neuro-immune and neuro-immune-endocrine interactions $(6,7)$.

The present article reviews our current understanding of the role of adrenergic innervation in bone resorption, an understanding based on the findings made by a variety of in vitro and in vivo studies. This article also reviews the role of $\beta$-blockers, used in clinical studies, for the prevention of bone fractures.

\section{Nerve-bone cell interplay}

In recent years, it has been demonstrated that human osteoblastic as well as osteoclastic cells are equipped with adrenergic receptors (ARs) and neuropeptide receptors $(1,2)$ and that they constitutively express diffusible axon guidance molecules known to function as a chemoattractant and/or chemorepellent for growing nerve fibers $(2,8)$. These findings suggest that the extension of axons of sympathetic and peripheral 
sensory neurons to osteoblastic and osteoclastic cells is required for the dynamic neural regulation of local bone metabolism. However, while several studies have shown a functional nerve-bone cell interplay, whether both osteoblastic and osteoclastic cells activation occurs as a direct response to neuronal activation or requires an intermediary cell is unclear. Therefore, we examined direct nerve-osteoblastic cell communication by using an in vitro co-culture model comprising mouse osteoblastic cells, MC3T3-E1, and neurite-spouting mouse superior cervical ganglia. Following loading with the calcium fluorophore Fluo-3, neurite-osteoblastic cell units were examined by confocal laser scanning microscopy. The addition of scorpion venom (SV) elicited neurite activation (i.e., $\mathrm{Ca}^{2+}$ mobilization) and, after a lag period, osteoblastic $\mathrm{Ca}^{2+}$ mobilization. The $\mathrm{SV}$ had no direct effect on the MC3T3-E1 cells in the absence of neurites. The addition of an $\alpha 1$-AR antagonist, prazosin, concentration-dependently prevented the osteoblastic activation that resulted as a consequence of the neural activation by SV. Thus, our recent findings demonstrate that MC3T3-E1 activation, as judged by $\mathrm{Ca}^{2+}$ mobilization, can be a direct consequence of contact with a specific activated nerve fiber. This evidence obtained in vitro demonstrates that nerve-osteoblastic cell cross-talk can occur in the absence of an intermediary transducing cell and that noradrenaline is an important mediator of this communication. Several recent in vivo and in vitro studies have demonstrated a sympathomimetic action on bone formation and resorption via osteoblastic and osteoclastic cells, respectively, expressing $\alpha$ - and $\beta$-ARs.

\section{Effect of adrenergic agonists on osteoclastogenesis}

Osteoclasts have a hematopoietic origin, and thus bone marrow culture techniques have been successfully employed to study the development of the osteoclasts from their precursor cells. Such cultures provide an appropriate system to investigate osteotrophic hormones, cytokines, and other bone-active factors that may be involved in the generation of osteoclasts. In this culture system, receptor activator of nuclear factor kappa B $(\mathrm{NF}-\kappa \mathrm{B})(\mathrm{RANKL})$ and osteoprotegerin (OPG) were reported to play an essential role in osteoclast differentiation. The expression of both proteins was shown to be regulated by several osteotrophic factors including $1,25(\mathrm{OH})_{2} \mathrm{D}_{3}$, interleukin (IL)- $1 \alpha$, IL-11, $\mathrm{PGE}_{2}$, transforming growth factor (TGF)- $\beta 1$, and PTH $(9-13) . \beta$ AR agonists, adrenaline and isoprenaline, have been also demonstrated to modulate osteoclastogenesis. The involvement of RANKL and/or OPG in adrenalineinduced bone resorption was shown by determining the effect of adrenaline on the mRNA expression of RANKL and OPG in MC3T3-E1 and on the formation of tartrate-resistant acid phosphatase (TRAP)-positive multinuclear cells (MNCs) in mouse bone marrow cultures, thus providing us a better understanding of the bone resorption induced by the sympathetic system (14). By use of the RT-PCR procedure, it was shown that the expression of RANKL and OPG mRNAs in osteoblastic cells was regulated by adrenergic stimulation, without the mediation of IL-11 and $\mathrm{PGE}_{2}$ produced in response to adrenaline (14). The expression of RANKL and OPG elicited by adrenaline appeared to be mediated by $\beta$ adrenergic and $\alpha$-adrenergic stimulation, respectively.

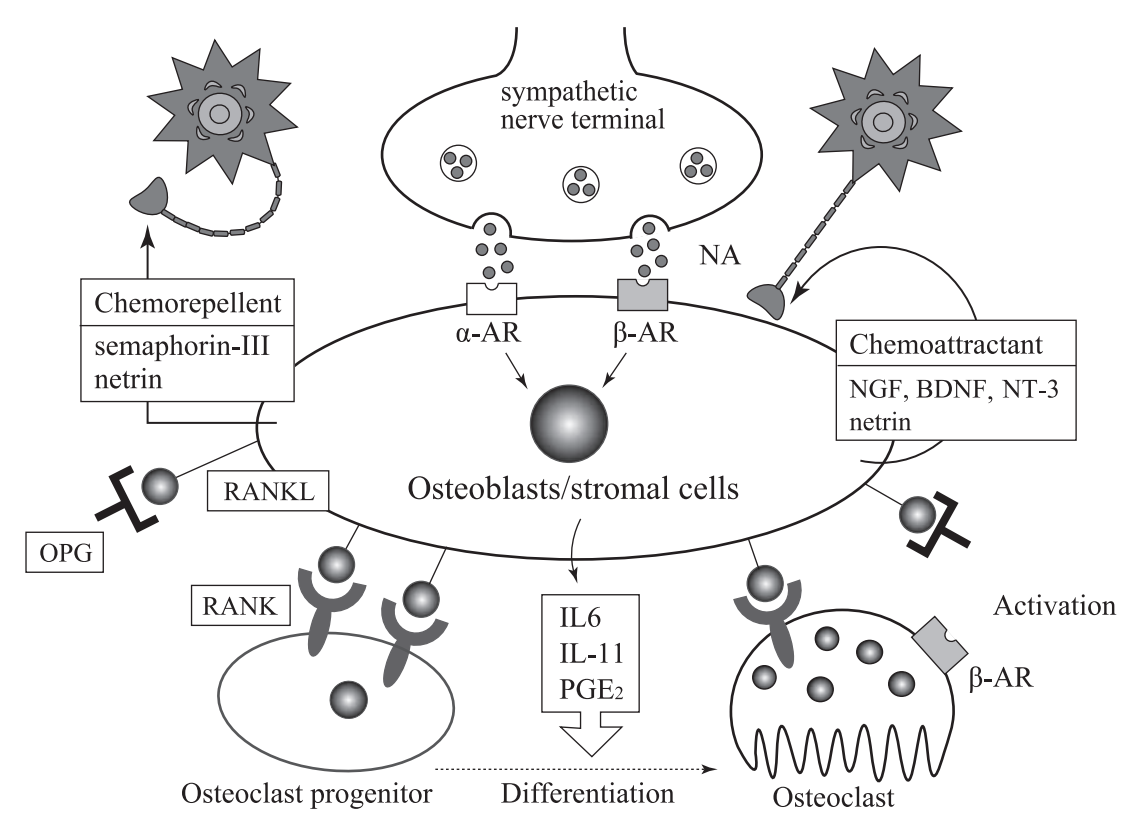

Fig. 1. Sympathomimetic action on osteoclastogenesis and osteoclastic activity. Both osteoblasts and osteoclasts constitutively express $\alpha 1 \mathrm{~B}-, \alpha 2 \mathrm{~B}-$, and $\beta 2$-ARs. Stimulation of $\beta 2$-ARs increases osteoclast formation and the expression of RANKL, IL-6, IL-11, and $\mathrm{PGE}_{2}$ in bone marrow or clonal osteoblastic cells. The increase in osteoclast formation is inhibited by OPG treatment, suggesting the involvement of the RANKL-RANK system in osteoclastogenesis caused by stimulating $\beta 2$-ARs. The expression of RANKL and OPG in MC3T3-E1 cells is increased by stimulating $\beta 2$-ARs and $\alpha$-ARs, respectively. In human osteoclasts, stimulation of $\beta$-ARs activates osteoclastic activity. Thus, increased sympathetic activity stimulates osteoclast formation as well as osteoclastic activity through $\beta 2$-ARs. 
Treatment of mouse bone marrow cells with adrenaline or isoprenaline generated TRAP-positive MNCs capable of excavating dentin to form resorptive pits on dentine slices and caused an increase in RANKL and a decrease in OPG production by the marrow cells (15). The osteoclast formation was significantly inhibited by OPG, suggesting the involvement of the RANKL-RANK system. Since the osteoclastogenesis in mouse bone marrow cells was not stimulated by an $\alpha$-AR agonist, the osteoclastogenesis may be regulated by the balance between RANKL and OPG production in osteoblasts /stromal cells. A possible mechanism for adrenergic stimulation of osteoclastogenesis is presented schematically in Fig. 1.

\section{Effects of adrenergic agonists on osteoclastic activity}

AR agonists stimulated cAMP synthesis in neonatal mouse calvariae and bone resorption in the presence of a phosphodiesterase inhibitor and an antioxidant (4). The cAMP synthesis stimulated by a $\beta$-AR agonist was inhibited by propranolol in bone organ cultures (16). The $\beta$-adrenergic stimulation of bone resorption might be mediated directly by activated osteoclasts and osteoclastogenesis enhanced by osteotrophic factors released from osteoblasts. In human osteoclast-like multinucleate cells constitutively expressing $\alpha 1 \mathrm{~B}-, \alpha 2 \mathrm{~B}-$, and $\beta 2$-ARs, $\beta$-AR agonists upregulated the expression of characteristic markers of the mature osteoclast such as integrin, carbonic anhydrase II, and cathepsin K; increased osteoclastic bone-resorbing activity; and clearly caused actin ring formation (17). However, these effects were not obtained by treatment with $\alpha$-AR agonists. These findings suggest that $\beta$-AR agonists directly stimulate boneresorbing activity in mature osteoclasts. In a clonal cell line of human osteoclast precursors (FLG 29.1 cells), catecholamines were also demonstrated to act as inducers of osteoclast maturation in vitro and as stimulators of osteoclast activity via the binding to $\beta 2$-ARs (18). As osteoclastogenesis-enhancing osteotrophic factors produced by $\beta$-adrenergic stimulation, IL-6, IL-11, and $\mathrm{PGE}_{2}$ were detected in human and mouse osteoblastic cells $(14,19)$. The co-induction of IL-6 and IL-11 by activation of $\beta$-ARs, which appears to be a common feature in osteoblastic cells, has been shown to be probably mediated via a common signal pathway involving the PKA and p38 MAPK systems, leading to the transcriptional activation of AP-1 in human osteoblastic cells. Thus, the $\beta$-adrenergic system could be involved in the catabolic effect of AR agonists on bone metabolism.

\section{Sympathetic activity toward bone resorption in vivo}

There are several lines of evidence showing that the sympathetic nervous system modulates bone resorption in vivo. Surgical removal of the superior cervical ganglion increased bone resorption (20), as did chemical treatment with guanethidine in newborn rats (21). However, in adult rats treated with guanethidine, bone resorption was conversely reduced (22). In rats sympathectomized in adulthood, Cherruau et al. (22) found a reduction in bone resorption and assumed that the inhibition reflected the acute effects of sympathectomy on their rats. By using a compartmentalization procedure, they showed that the resorption surface in the osteogenic compartment was reduced significantly in the guanethidine-treated rats, together with a fall in the number of osteoclasts and impaired osteoclast access to the bone surface. The effect on resorption by inhibiting preosteoclast differentiation and disturbing osteoclast activation by the acute sympathectomy suggest that depletion of sympathetic mediators could disturb osteogenic cell-mediated osteoclast differentiation. In addition, a sympathectomy-induced depletion of noradrenaline may be another possible mechanism for the reduction in bone resorption caused by sympathectomy. Such a mechanism is supported by several significant data showing the stimulation of bone resorption in a tissue culture system (4), an increase in preosteoclastic cell activity (18), and the stimulation of the synthesis of osteoclast-like cell formation-stimulating factors in osteoblastic cells by $\beta$-adrenergic stimulation $(14,19)$.

The intracerebroventricular (i.c.v.) injection of lipopolysaccharide (LPS), which is an inflammatory stimulus in the brain, was demonstrated to increase the output from the peripheral sympathetic nervous system (23, $24)$. To prove the physiological role of the sympathetic nervous system in bone metabolism in vivo, we performed RT-PCR to examine the effect of an i.c.v. injection of LPS on cyclooxygenase (COX)-2 and IL-6 mRNA expression in mouse calvaria $(23,24)$. The expression of both was increased by the injection. Both increases were inhibited by treatment with the neurotoxin 6-hydroxydopamine (6-OHDA) or by a $\beta$-blocker. Similarly, restraint stress induced the expression of IL-6 mRNA in mouse calvaria (24). This induction was not influenced by 6-OHDA, but was inhibited by propranolol. In addition, the treatment of calvaria with isoprenaline or noradrenaline increased $\mathrm{PGE}_{2}$ and IL-6 synthesis in an organ culture system. These findings show that the increase in gene expression caused by a restraint stress or i.c.v. injection of LPS was mediated by the activation of sympathetic nerve fibers and $\beta$-ARs in mouse calvaria and suggest that in vivo activation 


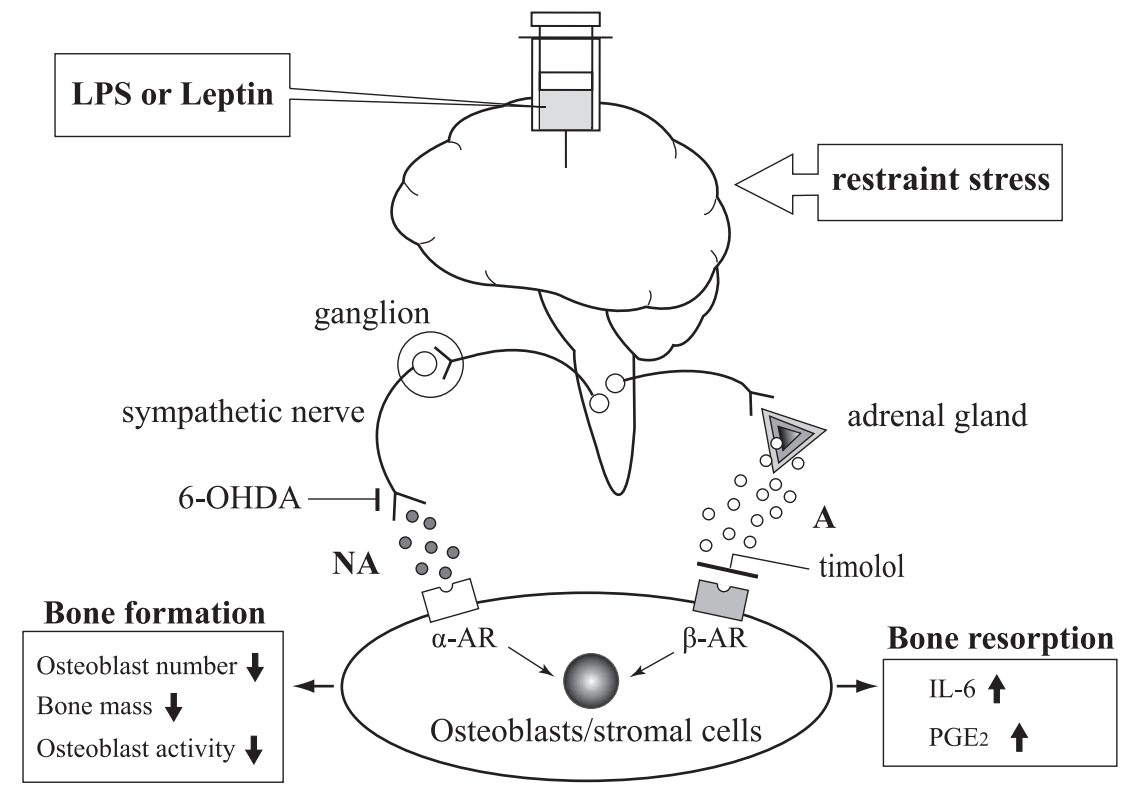

Fig. 2. Bone metabolism modulated by sympathetic nervous system. Central activation of the peripheral sympathetic nervous system caused by the intracerebroventricular (i.c.v.) injection of lipopolysaccharide (LPS) or leptin or by restraint stress alters bone formation and bone resorption. Centrally mediated activation of the sympathetic nervous system is associated with increased catecholamine levels. Leptininduced sympathetic activation has been reported to decrease bone formation via osteoblastic activity. On the other hand, either LPSor stress-induced activation increases the synthesis of osteoclastogenetic factors such as IL-6, and $\mathrm{PGE}_{2}$, which are factors that promote bone resorption. of the sympathetic nervous system modulates bone resorption. The above-mentioned findings are strongly supported by recent experiments (25) showing that $\beta 2$ AR-deficient $\left(A d r b 2^{-/-}\right)$mice had a more severe high bone mass phenotype than ob/ob or wild-type mice receiving $\beta$-blockers and that long-term leptin i.c.v. infusion did not reduce the bone mass of $A d r b 2^{-/-}$mice. Furthermore, the group just cited showed that the sympathetic nervous system favored bone resorption by increasing the expression of RANKL and that isoprenaline enhanced the generation of osteoclasts when wildtype, but not $A d r b 2^{-/-}$, osteoblasts were co-cultured with wild-type bone marrow macrophages.

Thus, these in vivo experiments modulating peripheral sympathetic nervous activity suggest that increased sympathetic nervous system activity leads to increased bone resorption through $\beta 2$-ARs (Fig. 2).

\section{Concluding remarks}

Both in vitro and in vivo experimental studies indicate that $\beta$-blockers may be effective toward osteoporosis attributed to increased sympathetic nervous activity. Inhibition of sympathetic nervous activity by $\beta$-blockers to inhibit bone resorption and/or to stimulate bone formation could, therefore, be an important new therapeutic avenue to cure osteoporosis. In a populationbased, case-control study generating data on adult women (26), men, and young women (27), the current use of $\beta$-blockers was demonstrated to be associated with a reduced risk of fractures, taken alone as well as in combination with thiazide diuretics. Thus, $\beta$-blockers generally do cause a reduction in bone fracture risk and higher bone mineral density. Another prospective study, however, showed no association between $\beta$-blocker use and fracture risk in perimenopausal and older women $(28-30)$. Therefore, there is currently no convincing evidence supporting the hypothesis that pharmacological blockade of the $\beta$-adrenergic system is beneficial to the human skeleton after menopause. Although $\beta$ adrenergic stimulation can be proposed as one of the causes of osteoporosis in experimental studies, the clinical usefulness of $\beta$-blockers on fracture risk must be analyzed in several patients with increased sympathetic nervous activity. Specifically, it is important to find a difference between users and nonusers with increased sympathetic tone. Further experimental and clinical studies are desirable to demonstrate the usefulness of $\beta$ blockers for the treatment of osteoporosis, by measuring markers of sympathetic nervous activity and bone metabolic activity, and are also needed to find $\beta$ blockers with high affinity for bone tissues.

\section{Acknowledgments}

Our studies mentioned in this review were partly supported by a grant-in-aid from the AGU High-Tech Research Center Project for Private Universities, with a matching fund subsidy from the Ministry of Education, Culture, Sports, Science, and Technology (MEXT: 2003-2007), and by a grant-in-aid from MEXT (17591956 to A.T.). 


\section{References}

1 Togari A, Arai M, Mizutani S, Mizutani S, Koshihara Y, Nagatsu T. Expression of mRNAs for neuropeptide receptors and $\beta$-adrenergic receptors in human osteoblasts and human osteogenic sarcoma cells. Neurosci Lett. 1997;233:125-128.

2 Togari A. Adrenergic regulation of bone metabolism: Possible involvement of sympathetic innervation of osteoblastic and osteoclastic cells. Microsc Res Tech. 2002;58:77-84.

3 Kellenberger S, Muller K, Richener H, Bilbe G. Formoterol and isoproterenol induce $\mathrm{c}$-fos gene expression in osteoblast-like cells by activating $\beta 2$-adrenergic receptors. Bone. 1998;22:471478.

4 Moore RE, Smith CKII, Bailey CS, Voelkel EF, Tashjian AH. Characterization of beta-adrenergic receptors on rat and human osteoblast-like cells and demonstration that beta-receptor agonists can stimulate bone resorption in organ culture. Bone Mineral. 1993;23:301-315.

5 Lerner UH. Neuropeptidergic regulation of bone resorption and bone formation. J Musculoskelet Neuronal Interact. 2002;2:440 447.

6 Opp MR, Imeri L. Sleep as a behavioral model of neuro-immune interactions. Acta Neurobiol Exp. 1999;59:45-53.

7 Aller MA, Arias JL, Lorente L, Nava MP, Duran HJ, Arias J. Neuro-immune-endocrine functional system and vascular pathology. Med Hypotheses. 2001;57:561-569.

8 Togari A, Mogi M, Arai M, Yamamoto S, Koshihara Y. Expression of mRNA for axon guidance molecules, such as semaphorin-III, netrins and neurotrophins, in human osteoblasts and osteoclasts. Brain Res. 2000;878:204-209.

9 Yasuda H, Shima N, Nakagawa N, Yamaguchi K, Mochizuki S, Yano K, et al. Identity of osteoclastogenesis inhibitory factor (OCIF) and osteoprotegerin (OPG): a mechanism by which OPG/OCIF inhibits osteoclastogenesis in vitro. Endocrinology. 1998;139:1329-1337.

10 Gao YH, Shinki T, Yuasa T, Kataoka-Enomoto H, Komori T, Suda T, et al. Potential role of cbfa1, an essential transcriptional factor for osteoblast differentiation, in osteoclastogenesis: regulation of mRNA expression of osteoclast differentiation factor (ODF). Biochem Biophys Res Commun. 1998;252:697702.

11 Hofbauer LC, Dunstan CR, Spelsberg TC, Riggs BL, Khosla S. Osteoprotegerin production by human osteoblast lineage cells is stimulated by vitamin D, bone morphogenetic protein-2, and cytokines. Biochem Biophys Res Commun. 1998;250:776-781.

12 Horwood NJ, Elliott J, Martin TJ, Gillespie MT. Osteotropic agents regulate the expression of osteoclast differentiation factor and osteoprotegerin in osteoblastic stromal cells. Endocrinology. 1998;139:4743-4746.

13 Murakami T, Yamamoto M, Ono K, Nishikawa M, Nagata N, Motoyoshi $\mathrm{K}$, et al. Transforming growth factor- $\beta 1$ increases mRNA levels of osteoclastogenesis inhibitory factor in osteoblastic/stromal cells and inhibits the survival of murine osteoclast-like cells. Biochem Biophys Res Commun. 1998; 252:747-752.

14 Takeuchi T, Tsuboi T, Arai M, Togari A. Adrenergic stimulation of osteoclastogenesis mediated by expression of osteoclast differentiation factor in MC3T3-E1 osteoblast-like cells. Biochem Pharmacol. 2001;61:579-586.

15 Ishizuka K, Hirukawa K, Nakamura H, Togari A. Inhibitory effect of CGRP on osteoclast formation by mouse bone marrow cells treated with isoproterenol. Neurosci Lett. 2005;379:47-51.

16 Majeska RJ, Minkowitz B, Bastian W, Einhorn TA. Effects of $\beta$ adrenergic blockade in an osteoblast-like cell line. J Orthop Res. 1992;10:379-384.

17 Arai M, Nagasawa T, Koshihara Y, Yamamoto S, Togari A. Effects of $\beta$-adrenergic agonists on bone-resorbing activity in human osteoclast-like cells. Biochimica Biophysica Acta. 2003;1640:137-142.

18 Frediani U, Becherini L, Lasagni L, Tanini A, Brandi ML. Catecholamines modulate growth and differentiation of human preosteoclastic cells. Osteoporosis Int. 1996;6:14-21.

19 Kondo A, Mogi M, Koshihara Y, Togari A. Signal transduction system for interleukin- 6 and interleukin-11 synthesis stimulated by epinephrine in human osteoblasts and human osteogenic sarcoma cells. Biochem Pharmacol. 2001;61:319-326.

20 Sandhu HS, Herskovits MS, Singh IJ. Effect of surgical sympathectomy on bone remodeling at rat incisor and molar root sockets. Anat Rec. 1987;219:32-38.

21 Hill EL, Turner R, Elde R. Effects of neonatal sympathectomy and capsaicin treatment on bone remodeling in rats. Neurosci. 1991;44:747-755.

22 Cherruau M, Facchinetti P, Baroukh B, Saffar JL. Chemical sympathectomy impairs bone resorption in rats: a role for the sympathetic system on bone metabolism. Bone. 1999;25:545551.

23 Inoue H, Kondo A, Togari A. Activation of the peripheral sympathetic nervous system increased the expression of cyclooxygenase-2 (COX-2) mRNA in mouse calvaria. Neurosci Lett. 2003;338:37-40.

24 Kondo A, Togari A. In vivo stimulation of sympathetic nervous system modulates osteoblastic activity in mouse calvaria. Am J Physiol Endocrinol Metab. 2003;285:E661-E667.

25 Elefteriou F, Ahn JD, Takeda S, Starbuck M, Yang X, Liu X, et al. Leptin regulation of bone resorption by the sympathetic nervous system and CART. Nature. 2005;434:514-520.

26 Pasco JA, Henry MJ, Sanders KM, Kotowicz MA, Seeman E, Nicholson GC. $\beta$-Adrenergic blockers reduce the risk of fracture partly by increasing bone mineral density: Geelong osteoporosis study. J Bone Miner Res. 2004;19:19-24.

27 Schlienger RG, Kraenzlin ME, Jick SS, Meier CR. Use of $\beta$ blockers and risk of fractures. JAMA. 2004;292:1326-1332.

28 Rejnmark L, Vestergaard P, Kassem M, Christoffersen BR, Kolthoff N, Brixen K, et al. Fracture risk in perimenopausal women treated with beta-blockers. Calcif Tissue Int. 2004;75: 365-372.

29 Levasseur R, Marcelli C, Sabatier JP, Dargent-Molina P, Breart G. Beta-blocker use, bone mineral density, and fracture risk in older women: results from the epidemiologie de l'osteoporose prospective study. J Am Geriatr Soc. 2005;53:550-552.

30 Reid IR, Gamble GD, Grey AB, Black DM, Ensrud KE, Browner WS, et al. $\beta$-Blocker use, BMD, and fractures in the study of osteoporotic fractures. J Bone Miner Res. 2005;20:613618. 